\title{
A novel scheme for energy-efficient bridge layer in sensor-cloud
}

\author{
Nasr Musaed S. Almurisi, T. Srinivasulu \\ KU College of Engineering \& Technology, Kakatiya University, India
}

\begin{tabular}{l}
\hline Article Info \\
\hline Article history: \\
Received Sep 9, 2019 \\
Revised Nov 10, 2019 \\
Accepted Nov 24, 2019 \\
\hline
\end{tabular}

\section{Keywords:}

Bridge layer

Energy-efficient WSNs

IoT-based heterogeneous WSN

Sensor-cloud

Virtualization

\begin{abstract}
Recently, Sensor-Cloud has been widely utilized in various domains, providing real-time monitoring and remote observations. The sensory data is collected from different heterogeneous WSNs, uploaded to the cloud, virtualized, and served for many user applications. However, the survivability of the physical sensors is a challenge, where the nodes are battery-powered and must be utilized wisely. The need is to extend their lifetime and, thus, ensuring cloud functionality and user satisfaction. In this paper, we address the energy-efficiency of the physical sensors in the SensorCloud paradigm. We propose a new scheme based on layered architecture, in which data transmitted to the cloud through a multi-hop routing. The new scheme introduces a novel algorithm to define a set of nodes called the bridge layer, receiving data from the cluster-head-layer and forwards to the sink node layer. Nodes in the bridge layer are selected according to their final score defined based on their energy-efficiency and distance-efficiency as given by the algorithm. Thus, ensuring a robust layer that helps in reducing the transmission energy and extending overall network lifetime. Our simulation results show an improved performance of our scheme over the scenario without the bridge layer, in terms of several parameters we considered.
\end{abstract}

Copyright $\Subset 2020$ Institute of Advanced Engineering and Science. All rights reserved.

\section{Corresponding Author:}

Nasr Musaed S. Almurisi,

KU College of Engineering \& Technology,

Kakatiya University, Warangal, India.

Email: eng.nasralmurisi17@gmail.com

\section{INTRODUCTION}

The importance of wireless sensor network (WSN) [1] arises due to the number of applications that depends on sensor devices to interact with the physical environment and extract useful data which will be used later for precise decision making [2]. These applications include healthcare application [3], environment monitoring application [4], military applications [5], etc. Recent years witnessed the increase of sensor devices utilized in the IoT technology [6], where most of the IoT-based applications depend on the data collected by sensors for processing and controlling many activities in real-life scenarios [7, 8]. Unfortunately, the traditional WSNs still have several limitations [9], such as processors speed, memory storage, energy consumption, wireless communication range, resource-sharing, etc. These limitations can be overcome by integration WSN with Cloud Computing technology [10].

Cloud computing, on the other hand, has powerful processing units and large storage [11]. A shared pool, of high-capability resources (such as massive storage, high-performance computing, software services) are available on demands to various users (in form of IaaS, SaaS, or PaaS). The combination of WSN and cloud computing is known as Sensor-Cloud [12], which meets the growing demand of sensing services and applications, and overcomes several implementation issues (e.g., scalability, heterogeneity, storage, data sharing, processing capability, communication range, etc.). The Sensor Cloud architecture consists of three layers: the physical layer, the cloud layer, and the application layer as shown in Figure 1. 
The physical layer consists of heterogeneous WSNs that represent the main physical part of the Sensor-Cloud, which are, usually, deployed over many remote places. Generally, an IoT-based WSN contains a number of different kinds of sensing nodes (e.g., light sensor, motion sensor, accelerator sensor, an audio sensor, camera sensor, etc.) spatially distributed to take readings of environmental data and monitor the surrounding conditions. The main role of this layer is to collect the data from sensor nodes (by the cluster heads), aggregate this data, and send it to the sink node which, then, forwards the received data to the cloud layer. Cloud layer (also known as a virtualization layer) is responsible for storing the received data, creating virtual sensors, managing service provisioning, and provide users with required services. All the information about physical sensors and their status are stored in service templates in the cloud layer. Whenever a user sends a request requiring a virtual sensor, this request will be served based on the information provided by the service template. The application layer represents the end-users who require services or sensed data from the sensor-cloud. The remote user can $\log$ in to the web browser and start reading data of his virtual sensors, or control the at any time.

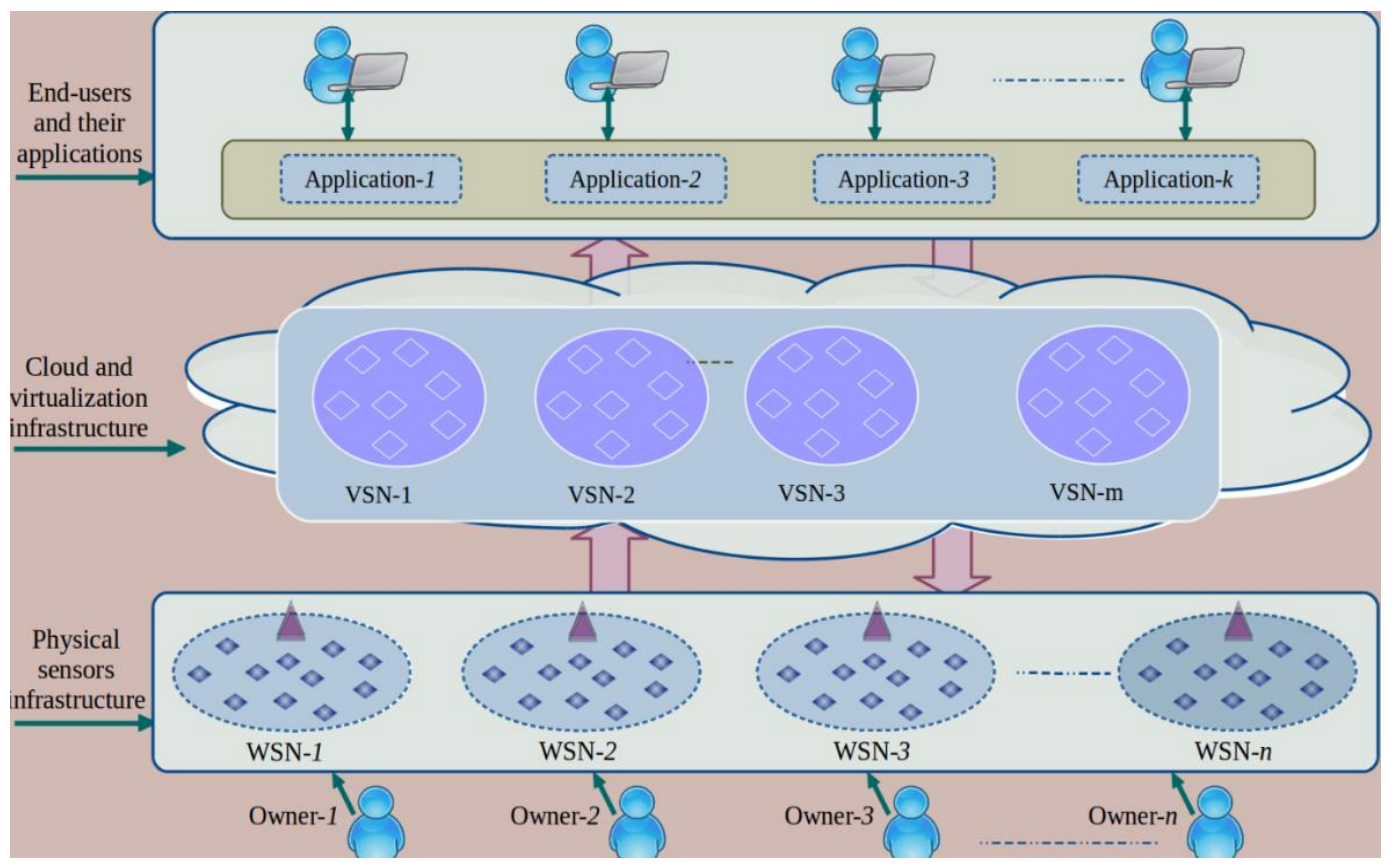

Figure 1. General reference view of the Sensor-Cloud

The recent advancement in the cloud-computing technologies and sensor network have led to developing several architectural and solutions for integrating WSN with cloud computing. In [13], an early architecture of sensor cloud is proposed, in which physical sensors can be managed using IT infrastructure. In [14] a new architecture is proposed for cloud-based heterogeneous sensing networks. The architecture is called Cloud4Sense', which provides two different models for resource management and integration: Data-centric model: provides the sensory data to various customers, and the Device-centric model: allows different customers to utilize particular virtual sensors as per their needs. In [15] ASCI (Agriculture SensorCloud Infrastructure) is proposed which consists of three layers: physical sensor layer, (ii) virtual sensor layer, and (iii) service cloud layer. The aim was to effectively process large amounts of data, provide different kinds of agricultural services, and enable a fast and reliable packet delivery to the destination in large-scale WSNs. In [16], a new sensor-cloud framework is proposed in order to facilitate building community-centric applications and enable connecting people, sensors, and software objects. In [17] OptiSeC (Optimal Sensor Cloud) is discussed that defines four main requirements for the sensor cloud, which are: security, efficiency, data management, and improved performance (e.g., robustness, scalability, routing, etc.).

Despite the fact that Sensor-Cloud can overcome certain limitations of WSN, there are still several challenges that need to be addressed such as energy consumption of the sensor nodes in the physical layer. Usually, the traditional cluster heads approach is being followed in the physical layer which consists of the following three layers: Sensing Layer, Cluster Heads, and Sink Layer as shown in Figure 2. In this approach sensor nodes in the sensing layer collect the data from the environment and forward it to the cluster heads 
which in turn aggregates the data and transmits it to the sink layer and from the sink layer to the cloud. The major drawback with this approach is that cluster heads consume most of their energy while transmitting the data to the sink node for longer distance without intermediate layers which in turn make the nodes expire early and the data transmission is ceased. This problem interrupts the process of creating and provisioning virtual sensors in the cloud and therefore the performance of Sensor-Cloud architecture is degraded. Hence it is mandatory to find out a suitable solution to increase the network lifetime and ensure that the performance of the sensor-cloud is optimal. Here we proposed a novel algorithm to reduce the energy consumption of sensor nodes during sending data towards the cloud. The proposed scheme introduces a new layer called (Bridge Layer). This layer acts as the intermediate layer between the cluster head layer and the sink layer and its purpose is to aggregate the data from several cluster heads and transmit it to the sink nodes. The proposed algorithm defines a set of robust nodes acting as a bridge layer which they are selected according to their final score defined based on their energy-efficiency and distance-efficiency. Through simulation, we compare the results of our scheme with the traditional Cluster Heads Layer(CHs Layer) and the results show significant improvement of network lifetime and the considerable amount of energy that is saved with the Bridge Layer.

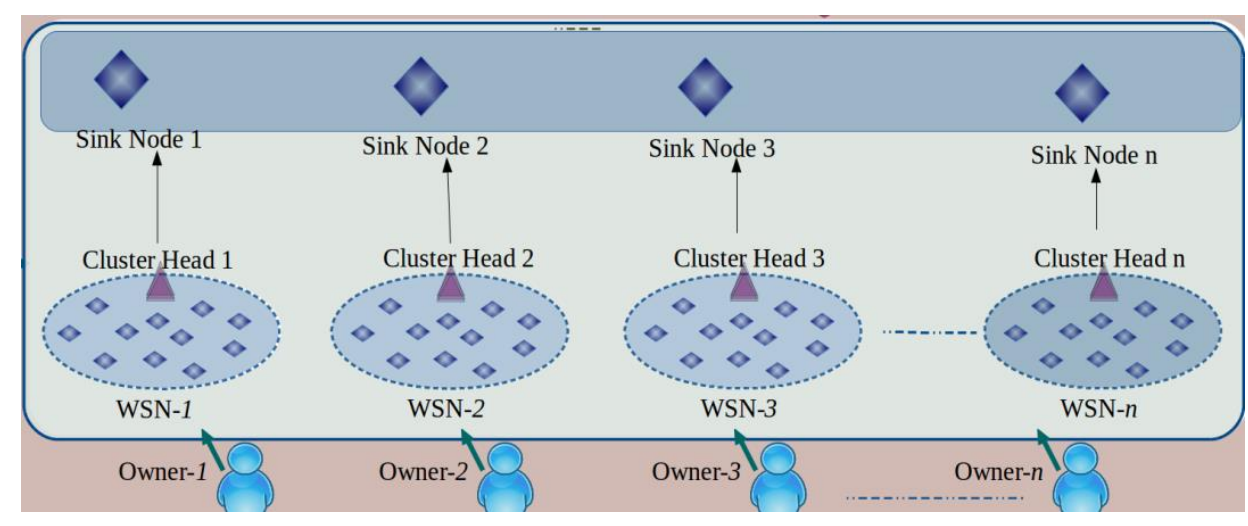

Figure 2. Cluster heads approach

\section{PROPOSED SCHEME}

In this section, we present a novel energy-efficient scheme for Sensor-Cloud that uses a multilayer architecture in which transmitting data from physical sensors to the cloud is based on a multi-hop routing. Our objective is to maximize the lifetime of nodes in the bottom layer by reducing the energy consumed during the data uploading process. The new algorithm defines a set of robust nodes acting as a bridge to the sink nodes and, thus, minimizing transmission energy expended by the sensor nodes in the physical layer.

\subsection{System Model}

The proposed scheme addresses the physical part of the Sensor-Cloud. The system model considered in the scheme consists of four layers namely: Sensing Layer, Cluster Heads Layer, Bridge Layer, and Sink Layer, as the model architecture is shown in Figure 3. The sensor nodes (SNs) in the first layer send their collected data to the cluster heads $(\mathrm{CHs})$ in the second layer $\mathrm{CHs}$, then aggregate the received data and transmit it upwards to the bridge nodes (BNs) in the third layer, which in turn, forward it directly to the sink nodes (SiNs) that is responsible for delivering data to the cloud. The nodes in the sink layer, are usually, have a continuous power supply and are not energy-limited. However, a number of key assumptions have been taken for the development of this scheme as listed below:

a) Sensor nodes are randomly distributed in the sensing area and are stationary once deployed (that are not movable).

b) SiNs in the sink layer is not energy-limited and, hence, have no constraints on their lifetime.

c) All the nodes have a power control so they can vary their transmission power according to the distance.

d) The network is a time-driven model, i.e., data is periodically available at the sensor nodes and need to be transferred to the cloud.

e) Energy-consumption is considered for data aggregation, transmission, and reception only. 


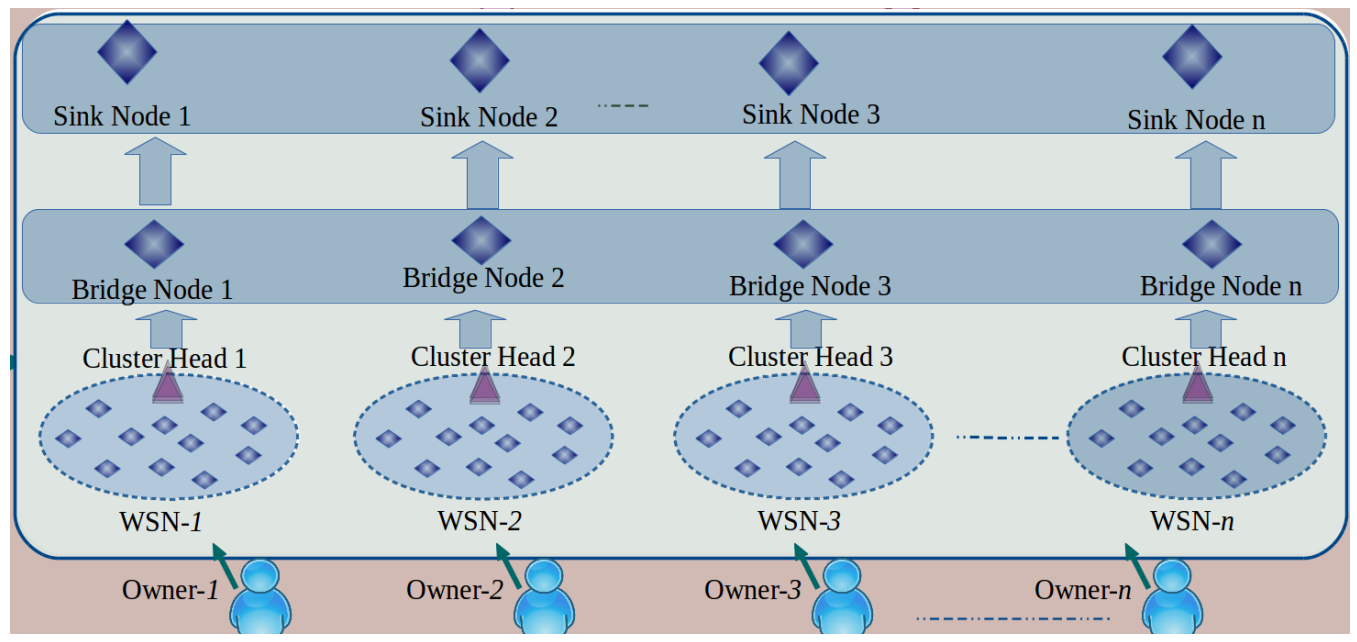

Figure 3. The architecture of the proposed system model

\subsection{Sensing Layer}

This layer consists of the physical heterogeneous sensor nodes that are dedicated to sense a variety of environmental parameters based on the network functionality and application requirements. Typically, in the Sensor-Cloud paradigm, several WSNs distributed in different targeted places are considered, forming heterogeneous sensing networks connected to the same cloud. These networks are responsible for collecting the environmental data required by the cloud which in turn serves many remote user applications. Thus, the survivability of these networks is important in order to ensure the cloud functionality and user satisfaction. However, the sensor nodes in this layer are, typically, battery-powered and it is difficult to replace their battery or recharge them, especially in the scenarios of dense networks or harsh environments. Therefore, we address this problem faced in this layer and introduce our algorithm for saving their energy and extend their lifetime.

\subsection{CHs Layer}

Cluster heads $(\mathrm{CH})$ in Sensor-Cloud schemes are a set of sensor nodes selected periodically in each transmission round based on some predefined criteria. $\mathrm{CHs}$ receive data from the sensor nodes, aggregate it, and forward it to the upper layers towards the cloud. Our algorithm for selecting bridge nodes can be applied to multi-levels heterogeneous WSNs, where the CHs election model is selected based on the heterogeneity level. The election model of SEP protocol [18] can be used for the $\mathrm{CH}$ election in the two-level heterogeneous network model, while the election model of EH-mulSEP protocol [19] can be used for $\mathrm{CH}$ election in network models with higher levels of heterogeneity. In both models, the CHs are selected based on two main parameters: weighted election probability and weighted election threshold, according to the initial energy of nodes where the nodes that have higher levels of energy become CHs more frequently than those having lower levels. For the purpose of evaluation, we have considered two levels of heterogeneity and use the SEP election model in our experimental simulation. In SEP protocol, two types of nodes are considered: first level ( $(\boldsymbol{v} \boldsymbol{l} \boldsymbol{l})$ and second level $(\boldsymbol{l v} \boldsymbol{l} \mathbf{2})$, where nodes in the latter have an additional amount of energy. The election probability of both levels $\left(\boldsymbol{p}^{l v l 1} \& \boldsymbol{p}^{l v l 2}\right)$ are defined as follows:

$$
\begin{aligned}
& \mathrm{p}^{\mathrm{lvl} 1}=\frac{\mathrm{p}^{\mathrm{opt}}}{1+\alpha \times \mathrm{m}} \\
& \mathrm{p}^{\mathrm{lvl} 2}=\frac{\mathrm{p}^{\mathrm{opt}} \times(1+\alpha \times \mathrm{m})}{1+\alpha \times \mathrm{m}}
\end{aligned}
$$

and the election thresholds $\left(\boldsymbol{T}^{l v l 1} \& \boldsymbol{T}^{l v l 2}\right)$ are given as:

$$
T_{S}^{l v l 1}=\left\{\begin{array}{rr}
\frac{p^{l v l 1}}{1-p^{l v l 1} \times\left(r \bmod \frac{1}{p^{l v l 1}}\right)}, \text { if } s \in G^{l v l 1} \\
0 \quad, \quad \text { otherwise }
\end{array}\right.
$$




$$
T_{S}^{l v l 2}=\left\{\begin{array}{rr}
\frac{p^{l v l 2}}{1-p^{l v l 2} \times\left(r \bmod \frac{1}{\left.p^{l v l 2}\right)}\right.}, \text { if } s \in G^{l v l 2} \\
0 \quad, \quad \text { otherwise }
\end{array}\right.
$$

where $\alpha$ and $\mathrm{m}$ are the extra energy and percentage of the $l v l l, l v l 2$ nodes respectively, $\mathrm{r}$ is the number of the present transmission round, $\mathrm{p}^{\text {opt }}$ is the optimal number of $\mathrm{CHs}$ in every round, while $\mathrm{G}^{l v l 1}$ and $\mathrm{G}^{l v l 2}$, are the set of nodes that have not been selected as $\mathrm{CHs}$ in the last $1 / p^{l v l 1} \& 1 / p^{l v l 2}$ rounds respectively

\subsection{Bridge Layer}

Our new scheme provides a novel algorithm to define the bridge nodes (BNs) in this layer. $\mathrm{BNs}$ have the role of receiving data from the $\mathrm{CHs}$ layer and forwards to the SiNs layer. The aim is to reduce the dissipated energy of the $\mathrm{CH}$ nodes by shortening their transmission distance which has a great impact on the energy consumed during data transfer. Instead of transmitting data to the sink nodes(SiN) for a long distance, $\mathrm{CHs}$ send their data to the BNs layer which, in turn, forwards to the $\mathrm{SiN}$, and, thus saving a considerable amount of energy. In fact, the nodes in the bridge layer (i.e., BNs) are a subset of the already elected $\mathrm{CH}$ nodes but are highly capable to take the role of data forwarding on behalf of the other $\mathrm{CH}$ nodes. We call this subset of nodes as the robust nodes and represented as the bridge layer in the architecture as shown in Figure 3. For selecting BN nodes, we define two key parameters: Energy Efficiency $\left(\mathrm{E}_{\mathrm{ef}}\right)$ and Distance Efficiency $\left(D_{\text {ef }}\right)$, which must be calculated for all the selected $\mathrm{CH}$ nodes according to the following formulas:

$$
\begin{aligned}
& E_{e f}=\frac{E r}{E r_{a v}} \\
& D_{e f}=\frac{D_{S i N}+D_{C H}}{2}
\end{aligned}
$$

Where $\mathrm{Er}_{\mathrm{av}}$ is the residual energy of the node and $\mathrm{Er}_{\mathrm{av}}$ is the average residual energy of all the nodes; while $\mathrm{D}_{\mathrm{SiN}}$ and $\mathrm{D}_{\mathrm{CH}}$ are functions in the node distance to the SiNs and to other CHs respectively, and are calculated as follows:

$$
\begin{aligned}
& D_{S i N}=\frac{D_{S N}}{D_{S N_{a v}}} \\
& D_{C H}=\frac{D_{C H_{\text {mean }}}}{D_{C H_{\text {mean }} a v}}
\end{aligned}
$$

Here $D_{S N}$ is the distance of the current node to the nearest $\mathrm{SiN}$ node and $D_{S N_{a v}}$ is the average distance of all the nodes to their SiN nodes, while $D_{C H_{\text {mean }}}$ is the mean distance of the current node to to all the cluster heads and $D_{C H_{\text {mean }} \text { av }}$ is the average of all the calculated $D_{C H_{\text {mean }}}$. After calculating $E_{e f}$ and $D_{e f}$ for all the selected $\mathrm{CHs}$, the algorithm find the final score Chscore for each of them according to the follwing formula:

$$
C H_{\text {score }}=E_{\text {ef }} \times w 1+D_{e f} \times w 2
$$

where $w 1$, and $w 2$ are scoring weights given by the administrator based on some predefined requirements. All the $\mathrm{CHs}$ nodes, then, are sorted decreasingly according to their final score, and only the top $\mathrm{k} \%$ nodes (i.e., the robust nodes) are selected as BNs layer nodes, where $\mathrm{k} \%$ is a positive integer representing the fraction of BNs to be selected from $\mathrm{CHs}$. This process runs at the setup phase in every transmission round directly after the $\mathrm{CH}$ election. As can be seen from the formulas above, we have focused on the transmission distance of nodes. This due to its higher influence on the energy consumed during sending data packets.

Typically, transmitting data for a short distance (i.e., LOS model) needs a small amount of energy, while transmitting data for larger distances (i.e., NLOS model) requires much higher energy which exponentially increases and obstacles usually exist [20]. However, the proposed scheme tries to minimize the total energy consumed by $\mathrm{CHs}$ which are periodically elected, and, hence, saving energy and extending the total lifetime of the network. 


\section{RESULTS AND ANALYSIS}

In this section, we evaluate the performance of the proposed algorithm for sensor-cloud architecture We have implemented our algorithm using MATLAB platform, and use the first-order energy dissipation model as have been used in [21, 18, 19]. This model considers the energy consumed for transmission, reception, and aggregation only, while the other energy demands are small and negligible. The amount of energy consumption at the transmitter $\left(E_{T X}\right)$ and receiver $\left(E_{R X}\right)$ is given as follows:

$$
\begin{aligned}
& E_{T X}= \begin{cases}l_{b} \times\left(E_{T X-\text { elec }}+E_{f s} \times d^{2}\right), \\
l_{b} \times\left(E_{T X-\text { elec }}+E_{m p} \times d^{4}\right)\end{cases} \\
& E_{R X}=l_{b} \times E_{R X-\text { elec }} \text { otherwise }
\end{aligned}
$$

Where $E_{f s}$ and $E_{m p}$ are the free space and multipath amplifier models respectively used according to the transmission distance $\mathrm{d}$, where a given threshold $d_{0}$ is defined as follows:

$$
d_{0}=\sqrt{E_{f s} / E_{m p}}
$$

\subsection{Experimental Setup}

The network setup and the parameters used during simulation are configured as shown Table 1. We evaluate the efficiency of our algorithm in comparison with the scenario in which no bridge nodes are defined.

Table 1. Parameter Values

\begin{tabular}{cc}
\hline Parameter & Values \\
\hline Deployment Area & $200 \mathrm{~m} \mathrm{X} 200 \mathrm{~m}$ \\
Number of Nodes & 1000 \\
Number of heterogeneity levels & 2 \\
$\alpha$ & 2 \\
$\mathrm{~m}$ & 0.4 \\
$l_{b}$ & 4000 \\
$p^{o p t}$ & 0.15 \\
$k$ & $0.33 \mathrm{X} \mathrm{P}$ opt \\
$E_{0}$ & 0.5 \\
$E_{T X-e l e c}$ & $50 \mathrm{~nJ} / \mathrm{bit}$ \\
$E_{R X-e l e c}$ & $50 \mathrm{~nJ} / \mathrm{bit}$ \\
$E_{a m p}$ & $0.0013 \mathrm{pJ} / \mathrm{bit} / \mathrm{m} 2$ \\
$E_{s f}$ & $10 \mathrm{pJ} / \mathrm{bit} / \mathrm{m} 2$ \\
$E_{d a}$ & $5 \mathrm{~nJ} / \mathrm{bit} / \mathrm{signal}$ \\
\hline
\end{tabular}

The number of considered parameters are listed:

a) Frist node dies (FND): defines the network stability period

b) Last node dies (LND): defines the overall network lifetime.

c) Half node die (HND): measures the network lifetime when $50 \%$ of the node have been expired.

d) Number of alive/dead nodes over the simulation time.

e) Amount of consumed/dissipated energy over the simulation time.

f) Number of messages sent towards cloud from sensor nodes during simulation time.

\subsection{Result Analysis}

Table 2 presents the network lifetime measures (i.e., FND, HND, and LND) for both scenarios. We can see that the bridge layer added to the network leads to an improvement in these three measures over the traditional case in which only the $\mathrm{CHs}$ layer is considered. The network stability period is improved by $102 \%$, while the overall network lifetime is extended by $36 \%$. This indicates that our new scheme has a positive impact on the energy-efficiency where the nodes consume less amount of energy during data transmission from sensing from $\mathrm{CHs}$ layers towards the sink layer. 
Table 2. Network Live-time Measure

\begin{tabular}{cccc}
\hline $\begin{array}{c}\text { Network Lifetime } \\
\text { Measure }\end{array}$ & \multicolumn{2}{c}{$\begin{array}{c}\text { Value in Terms of Rounds } \\
\text { CHs Layer }\end{array}$} & $\begin{array}{c}\text { Bain Achieved } \\
\text { Bridge Layer }\end{array}$ \\
\hline FND & 344 & 696 & 102 \\
HND & 1336 & 1427 & 07 \\
LND & 4000 & 5446 & 36 \\
\hline
\end{tabular}

Figures 4 and 5 show the number of alive and dead nodes during the simulation lifetime respectively. From this, it is clear that the nodes expire slowly compared to the traditional scenario. Here, the bridge nodes selected in the new schemes we introduced help in balancing energy-load among CHs in the second layer, and thus, leading to reduction in the amount of energy expended for transmitting data to short distances to bridge layer instead of transmitting to the sink layer for larger distance, which delay the nodes expiration.

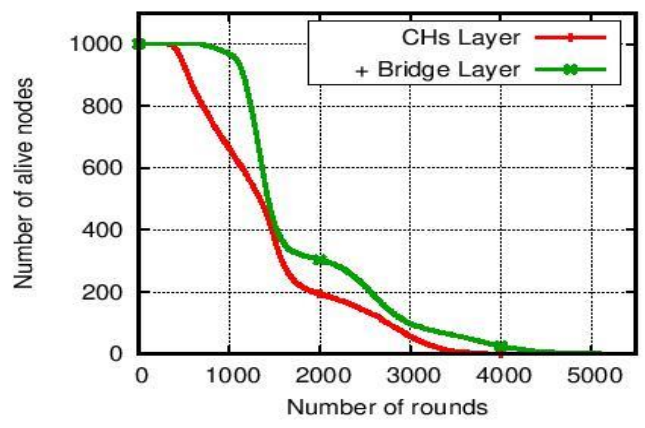

Figure 4. Alive nodes vs. Number of rounds

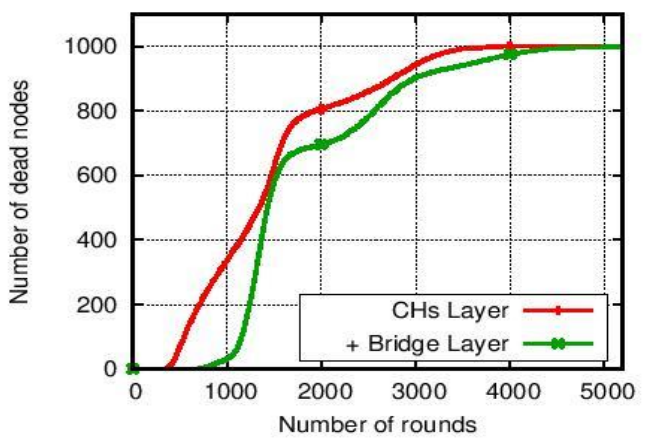

Figure 5. Dead nodes vs. number of rounds

In Figure 6, we present the amount of energy with respect to the simulation time, which show that nodes in our scheme has less energy demand during the whole network operation as a result of reduced transmission distance and hence a significant amount of energy is saved. such a significant increase in the network lifetime and energy saved is important in order to ensure extended services provided by the network through the cloud where more data is reported to the cloud than traditional schemes. In Figure 7, we show the number of messages sent by the nodes in the first layer of the network (i.e., sensing layer) to the cloud through intermediate layers, with a total increase equals to $26 \%$.

However, The results we discussed above indicate that the bridge layer we introduced in our scheme has the advantage of reducing the total energy consumed by the physical sensor nodes and therefore leading to extended network lifetime, and increased data reported to the cloud, which then can be virtualized and served to many users for a longer time.

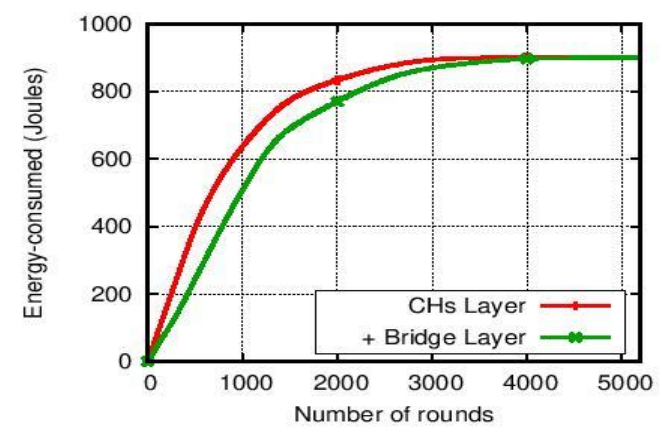

Figure 4. Amount of energy dissipated during simulation

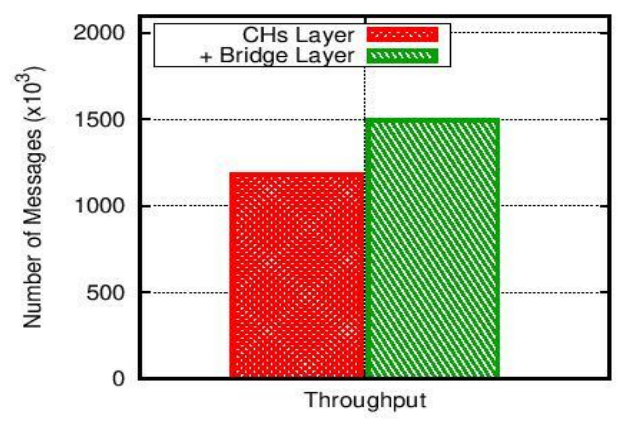

Figure 5. Total number of messages transmitted 


\section{CONCLUSION}

In this paper, we have introduced a new scheme for data routing from the physical layer to the cloud in Sensor-Cloud technology. A new algorithm is proposed to select a number of nodes to take the role of a bridge layer that links cluster heads with the sink layer. The aim is to reduce the transmission distance of the cluster heads to save their energy, and, hence, helps in extending the overall network lifetime. We have discussed the new scheme in detail, presenting the main parameters and scoring formulas. We have simulated our algorithm in Matlab software and compared its performance with that of traditional routing and showed its superiority in terms of a number of parameters we used. Our future plan is to study using energyharvesting as an additional layer to show its impact on network performance.

\section{ACKNOWLEDGEMENTS}

This work is carried out at Kakatiya University College of Engineering and Technology in India, to rescieve PhD degree in Electronic and Communication Engineering.

\section{REFERENCES}

[1] I. Akyildiz, W. Su, Y. Sankarasubramaniam, and E. Cayirci, "Wireless sensor networks: a survey," Computer Networks, vol. 38, no. 4, pp. $393 \quad-\quad 422, \quad$ 2002. [Online]. Available: http://www.sciencedirect.com/science/article/pii/S1389128601003024

[2] A. S. H. Abdul-Qawy and T. Srinivasulu, "SEES: a scalable and energy-efficient scheme for green IoT-based heterogeneous wireless nodes," Journal of Ambient Intelligence and Humanized Computing, Springer, pp. 1-26, March 2018.

[3] N. Sghaier, A. Mellouk, B. Augustin, Y. Amirat, J. Marty, M. E. A. Khoussa, A. Abid, and R. Zitouni, "Wireles sensor networks for medical care services," in 2011 7th International Wireless Communications and Mobile Computing Conference, July 2011, pp. 571-576.

[4] M. T. Lazarescu, "Design of a wsn platform for long-term environmental monitoring for iot applications," IEEE Journal on Emerging and Selected Topics in Circuits and Systems, vol. 3, no. 1, pp. 45-54, March 2013.

[5] H. B. Lim, D. Ma, B. Wang, Z. Kalbarczyk, R. K. Iyer, and K. L. Watkin, "A soldier health monitoring system for military applications," in 2010 International Conference on Body Sensor Networks, June 2010, pp. 246-249.

[6] G. Basavaraj and C. Jaidhar, "Low latency and energy efficient cluster based routing design for wireless sensor network," Indonesian Journal of Electrical Engineering and Computer Science, vol. 13, pp. 615-625, 022019.

[7] Y. S.ALDEEN and K. Qureshi, "New trends in internet of things, applications, challenges, and solutions," TELKOMNIKA (Telecommunication Computing Electronics and Control), vol. 16, pp.1114-1119, 062018.

[8] S. Sharma and S. Sebastian, "Iot based car accident detection and notification algorithm for general road accidents," International Journal of Electrical and Computer Engineering (IJECE), vol. 9, p. 4020, 102019.

[9] O. MAHDI, Y. Rafid, A. Ghazi, M. Mohammed, A. Wahid, M. Yamani, and I. Idris, "An energyaware and loadbalancing routing scheme for wireless sensor networks," Indonesian Journal of Electrical Engineering and Computer Science, vol. 12, pp. 1312-1319, 122018.

[10] S. Madria, V. Kumar, and R. Dalvi, "Sensor cloud: A cloud of virtual sensors," IEEE Software, vol. 31, no. 2, pp. 70-77, Mar 2014.

[11] R. Kaur and G. Kaur, "Proactive scheduling in cloud computing," Bulletin of Electrical Engineering and Informatics, vol. 6, pp. 174-180, 062017.

[12] A. Alamri, W. S. Ansari, M. M. Hassan, M. S. Hossain, A. Alelaiwi, and M. A. Hossain, "A survey on sensorcloud: Architecture, applications, and approaches," International Journal of Distributed Sensor Networks, vol. 9, no. 2, p. 18, 2013.

[13] M. Yuriyama and T. Kushida, "Sensor-cloud infrastructure - physical sensor management with virtualized sensors on cloud computing," in 2010 13th International Conference on Network- Based Information Systems, Sept 2010, pp. 1-8.

[14] M. Fazio and A. Puliafito, "Cloud4sens: a cloud-based architecture for sensor controlling and monitoring," IEEE Communications Magazine, vol. 53, no. 3, pp. 41-47, March 2015.

[15] K. Kim, S. Lee, H. Yoo, and D. Kim, "Agriculture sensor-cloud infrastructure and routing protocol in the physical sensor network layer," International Journal of Distributed Sensor Networks, vol. 10, no. 3, p. 437535, 2014.

[16] M. M. Hassan, B. Song, and E.-N. Huh, "A framework of sensor-cloud integration opportunities and challenges," in Proceedings of the 3rd International Conference on Ubiquitous Information Management and Communication, ser. ICUIMC '09. ACM, 2009, pp. 618-626.

[17] M. U. Atif and M. A. Shah, "Optisec: In search of an optimal sensor cloud architecture," in 23rd International Conference on Automation and Computing (ICAC), Sept 2017, pp. 1-6.

[18] G. Smaragdakis, I. Matta, and A. Bestavros, "SEP: A stable election protocol for clustered heterogeneous wireless sensor networks," in International Workshop on SANPA, 2004, pp. 251-261.

[19] A. S. H. Abdul-Qawy and T. Srinivasulu, "EH-mulSEP: Energy-harvesting enabled multi-level SEP protocol for IoT-based heterogeneous WSNs," in 3rd International Conference on Applied and Theoretical Computing and Communication Technology (iCATccT), Presented, December 2017. 
[20] A. S. H. Abdul-Qawy, N. M. S. Almurisi, A. P. Kumar, and T. Srinivasulu, "Major energy dissipation sources in the IoT-based wireless networks," 4th International Conference on New Frontiers of Engineering, Science, Management and Humanities (ICNFESMH-2017) and International Journal of Electronics, Electrical and Computational System (IJEECS), vol. 6, no. 9, pp. 155-161, 2017.

[21] W. B. Heinzelman, A. P. Chandrakasan, and H. Balakrishnan, "An application-specific protocol architecture for wireless microsensor networks," IEEE Transactions on Wireless Communications, vol. 1, no. 4, pp. 660-670, 2002.

\section{BIOGRAPHIES OF AUTHORS}

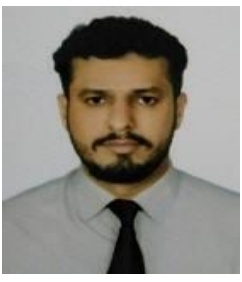

Nasr Musaed S Almurisi. (Research Scholar). He have a B.Tech degree in Computer Science and Engineering from JNTH Hyderabad, India. And M.Tech Computer Science and Engineering from JNTH Hyderabad, India. Currently, he is a Ph.D. research Scholar at college of engineering and technology in Kakatiya University, Warangal India. He published 5 reseach papers in international journals and conferences. He research interests are Internet of Things (IoT), virtualization, cloud computing, and wireless sensor networks.

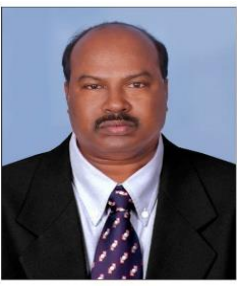

Prof. T. Srinivasulu (Guide \& Supervisor) is working as Professor and Dean, Faculty of Engineering and Technology, Kakatiya University, He obtained M. Tech from IIT Dhanbad, and Ph.D. from Kyushu University, Japan. He was completed Proficiency Courses and diplomas from IISc Bangalore, IIT Kharagpur, CEDTI Gorakhpur, NPA and NRDC New Delhi. The major administrative positions held are Principal, Director, General Manager, Coordinator, and Head of the Department. He published over 157 research papers in international journals and conferences. He is peer reviewer for Elsevier International Journals, ISOI Journal, and IEEE Access. He is editorial board member of IJAA, IJRI, IJECE, IJCSE, International Journal of Sensor and Sensor Networks, Science Publishing Group of USA. 